\title{
FUENTES PARA EL ESTUDIO DE LA ROMANIZACIÓN EN LA MESETA NORTE
}

\author{
Sources for the study of the romanization in the Plateau North \\ Javier PÉREZ MARTÍN \\ Universidad de Salamanca \\ E-mail: javi_4k@usal.es \\ Fecha de recepción: $18-01-2010$ \\ Fecha de aceptación: 29-01-2010
}

\begin{abstract}
RESUMEN: La conquista romana del interior peninsular modificará las formas de vida en este territorio a todos los niveles, en un proceso que la historiografía ha dado en llamar romanización y que durará más de dos siglos. Dentro de este contexto histórico y geográfico, nos centraremos en la importancia de las distintas fuentes, exponiendo ejemplos significativos de cada una de ellas con los que señalaremos la abundante información que nos aportan, los distintos métodos de trabajo que usamos al manejarlas y algunas de las dificultades que nos pueden surgir en su estudio. Todo ello con el objetivo de mostrar la necesidad del investigador en combinarlas y realizar un estudio detallado de las mismas, con el fin de conseguir una correcta interpretación del pasado.
\end{abstract}

Palabras Clave: Conquista romana; romanización; fuentes clásicas; numismática; epigrafía; filología; arqueología.

ABSTRACT: The Roman conquest of the peninsular interior will modify the forms of life in this territory to all the levels, in a process that the historiography has given in calling romanization and that will last more than two centuries. Inside this historical and geographical context, we will centre on the importance of the different sources exposing significant examples of each one of them with which we will indicate the abundant information that they us contribute, the different methods of work that we use on having handled them and some of the difficulties that us can arise in his study. All this with the aim to show the need of the investigator in a detailed study of the same ones combining and realizing them, in order to obtain a correct interpretation of the past. 
Keywords: Roman Conquest, Romanization, Classic Sources, Numismatic, Epigraphy, Philology, Archaeology.

En el presente trabajo, nos proponemos realizar una síntesis acerca de distintas fuentes que poseemos para estudiar las transformaciones que tendrán lugar con motivo de la conquista romana del interior peninsular. Estos cambios serán responsables de la nueva configuración social, política, económica y religiosa que caracterizará a este territorio durante la época imperial de la Hispania Romana.

Como motivo de la brevedad de está comunicación, consideramos que, mediante cuatro modelos característicos relacionados con nuestro tema a tratar, puede quedar clara la panorámica del tema a trabajar, intentando enfocarlo hacia cualquier persona que este interesada en este periodo en particular o se proponga realizar una futura investigación, pudiéndole servir esta comunicación como herramienta orientativa con la que comenzar a investigar las distintas fuentes concernientes a esta época.

Partiendo de esta idea hemos descrito brevemente las distintas fuentes con las que se trabaja en este periodo histórico, así como los recursos de los que nos valemos para ello. Para llevar a cabo este propósito utilizaremos, a modo de modelos, cuatro ejemplos significativos de cada una de las fuentes que se tratan, ubicándolas cada una de ellas en una zona diferente de la Meseta, teniendo como protagonista a un pueblo prerromano distinto en cada caso. Vamos a comenzar trabajando las fuentes clásicas relacionadas con el pueblo vettón, para posteriormente analizar un epígrafe del área astur, una moneda de la zona celtibérica y, por último, restos arqueológicos pertenecientes a una necrópolis vaccea. Mediante estos ejemplos se intenta mostrar el gran aporte de información que podemos extraer de cada fuente, los diferentes métodos de análisis empleados, las múltiples complicaciones con las que nos podemos encontrar en cada caso y la dificultad que supone realizar determinadas interpretaciones. En último lugar, realizaremos un repaso acerca de aspectos relacionados con la obtención de información bibliográfica, enumerando una serie de recursos que nos pueden facilitar la tarea de recopilar información, acerca de un determinado tema de estudio.

\section{FUENTES CLÁSICAS}

A la hora de estudiar las fuentes clásicas relacionadas con la romanización de la Meseta Norte, lo primero que se deshecha es la idea de imparcialidad respecto a los pueblos y los hechos que en ellas se narran. El origen, bajo órbita romana de la totalidad de sus autores, pone en evidencia el ca- 
rácter favorable hacia Roma con el que nos vamos a encontrar, que irá variando ligeramente en función del momento en que se redacten los textos, la intención de los autores, y la concepción sobre los pueblos meseteños por parte de los mismos.

Para nuestro primer apartado nos centraremos, como dijimos anteriormente, en las fuentes clásicas referentes al pueblo vettón, poniendo de manifiesto algunas de las claves más importantes para trabajar con fuentes clásicas.

En primer lugar, señalar que, en general, los autores clásicos que nos ofrecen referencias acerca de los vettones no son contemporáneos a los hechos y motivos que tratan. Para realizar sus escritos beberán de otros documentos, que habrán sido escritos bajo un contexto y unas condiciones determinadas, a las que debemos añadir además la propia intención del autor que los recopila. Es un factor clave conocer el contexto histórico en el que se escribió cada uno de los textos, para intentar sondear la intención que pudiera poseer el mismo. Por ello, nos parece interesante hacer un repaso sobre las diversas referencias relacionadas con el pueblo vettón, que nos han dejado dichos autores, mediante un orden cronológico que nos permita observar los aspectos en los que se centran y las diferentes pautas desde las que tratan el tema según cada época.

Los documentos más antiguos que tratamos son de época republicana. Se cuenta, para este periodo, con referencias acerca de los vettones en dos citas de Cornelio Nepote (110-99 a. C. - 24 a. C.) $)^{1}$, en algunos fragmentos de Livio (64-59 a.C. - 17 d.C. $)^{2}$, y en las narraciones de Cesar (100- 44 a. C. $)^{3}$. Estos textos obedecen a episodios bélicos relacionados con la conquista púnica y romana de la zona occidental de la península, pudiendo extraer de ellos el campo de acción de los vettones y su papel de aliado, con otros pueblos vecinos, en su lucha frente a Roma.

Podemos obtener una visión más amplia por parte de Estrabón (6463 a. C. -20 a. C.) en el tercer libro de su Geographia. En esta obra, para la cual utiliza escritos de Posidonio (135 - med. s. I a.C.), realiza una diferenciación geográfica de los pueblos que ocupan el interior meseteño, gracias a la cual podemos situar a los vettones entre los ríos Tajo y Duero ${ }^{4}$. Destaca también características de dichos pueblos al calificarlos como montañeses y considerarlos francamente atrasados, señalando determinadas costumbres, que califica como raras, para describirlos ${ }^{5}$.

Para la época imperial, contamos con los textos de Lucano (39 - 65 d. C.) y de Plinio el Viejo (23 - 79 d. C.), quienes, si bien residieron en la Península Ibérica, su aportación para nuestro conocimiento es muy desigual. Lucano, poeta cordobés, hará una mención al pueblo vettón en uno de sus versos, indiferenciándolo de los demás pueblos celtas ${ }^{6}$. Plinio, sin embargo, 
en su Naturalis Historia, sí realizará un análisis más formal acerca de los pueblos que componen la Hispania romana, situando a los vettones de nuevo en torno al Tajo ${ }^{7}$ y el río Duero ${ }^{8}$. Algunos de los datos geográficos de Plinio serán extraídos de referencias del general Agripa, recogiendo ya una nueva situación creada con el Principado, en el que los vettones aparecen mucho más centrados territorialmente en la Meseta Norte', trabajando un contexto en el que ya se ha producido una nueva reestructuración del territorio por parte de Roma. En las referencias de este autor, el contexto, tanto geográfico como el ideológico ha variado respecto a los autores anteriores, estando influidos los textos por nuevas realidades socio-políticas diferentes a las de la época republicana.

Las últimas fuentes clásicas a utilizar para este apartado serán las procedentes del siglo II d. C, personificadas en las figuras de Apiano y Ptolomeo.

Apiano (95 - 160 d. C.), en su Historia Romana, relatará los acontecimientos de la conquista y pacificación de la Península Ibérica. Entre su trabajo y los acontecimientos que trata, han pasado ya más de dos siglos, motivo éste que le hará basarse en numerosas fuentes a la hora de narrar dichos acontecimientos. En sus textos irá enlazando cronológicamente los acontecimientos sucedidos, valiéndose para ello de una fuente principal personificada en la figura de Polibio, al que su estancia en la península ibérica, durante las guerras celtíberas le sirvió para estudiar la geografía, los pueblos y las costumbres de Hispania. Suplirá las partes perdidas de éste con datos extraídos de un alto conglomerado de autores entre los que podemos citar a Salustio, Paulo Clodio, Posidonio, Livio, Salustio, Celio Antipatro, Jerónimo de Cardia, Julio César, Augusto, Asinio Polión, Plutarco, Diodoro.

Ptolomeo (96 - 168 d. C.), por su parte, recogerá una lista de lugares vettones (II 5, 6-7), donde aparecerán la ciudades de Lancia Oppidana Cottaeobriga, Salmantica, Augustobriga, Ocelum, Capara, Manliana, Lacimurga, Deóbriga, Obila y Lama, junto con sus respectivas coordenadas. Sin embargo, debemos tener en cuenta que no conocemos con exactitud ni el periodo histórico, ni las distintas fuentes en las que pudo documentarse a la hora de realizar dicha lista, además, debemos añadir los problemas suscitados a causa de la conquista y el ordenamiento del territorio realizado por parte de Roma, siendo por ello aventurado considerar si determinadas poblaciones fueron o no vettonas, y en que periodo. Algunas de las ciudades que tacha como vettonas, como es el caso de Capara aparecen en la cita de Plinio IV, 118 como estipendiarios de Lusitania, otras como Lacimurga son denominadas célticas (Plinio III, 14), en cuanto a Salmantica continua hoy en día el debate sobre su condición vaccea o vettona y de Deóbriga o Lama todavía se desconoce su localización. 
Después de este repaso, es clave destacar la importancia de ser cautelosos al utilizar los distintos textos clásicos que poseemos. El contexto histórico y geográfico tanto del autor del documento, como de los sucesos que narra nos permite crear unas pautas a la hora de analizar los mismos, fijándonos también en las intenciones, estatus social, y en los textos que usa a modo de fuentes, con el objetivo de conseguir extraer la mayor cantidad de información veraz de los escritos.

\section{FUENTES EPIGRÁFICAS}

En uno de sus artículos José Manuel Roldán Hervás destacará la epigrafía, como el grupo más interesante de fuentes escritas para el conocimiento de la época romana, "tanto por su carácter material, o vivo al que hay que conceder aún más valor que a las propias fuentes literarias ya que no está deformado por la tradición o por intereses ajenos a la verdad de tipo estético, propagandístico o moralista de que se resienten en gran parte de las fuentes clásicas" ${ }^{10}$.

La Epigrafía es una ciencia que posee una gran utilidad para la Historia de la Antigüedad, ya que tiene como objetivo el estudio de los textos antiguos escritos sobre material perdurable, siendo una disciplina eminentemente práctica pero que exige unos conocimientos previos. Para el estudio de la romanización del interior peninsular se suelen utilizar, en general, epígrafes procedentes mayoritariamente de época bajo-republicana e imperial. En ellos resulta interesante observar como las poblaciones meseteñas van a ir adquiriendo a lo largo del tiempo influencias romanas tanto en aspectos lingüísticos, como nominativos, administrativos, religiosos, jurídico o sociales.

Del Bronce de Bembibre (fig.I), se pueden extraer alguna de las causas responsables de la evolución de estas comunidades bajo órbita romana. Con su estudio obtenemos información acerca de diversas características de los pueblos prerromanos y sobre la política organizativa y fiscal que desarrollo Roma en el noroeste peninsular.

$\mathrm{Al}$ realizar en primer lugar un análisis externo de este epígrafe, situamos el hallazgo de este documento en un castro de los alrededores de Bembibre en 1999. Se trata de una placa de bronce rectangular, en buen estado de conservación que presenta un orificio en la parte superior que nos indicaría que el texto estuvo expuesto. El campo epigráfico se enmarca mediante dos líneas incisas paralelas, deduciéndose de las mismas que el escriba que grabo el texto se daría cuenta a partir del vigésimo segundo renglón de que había más espacio que el previsto, solucionándolo utilizando un número menor de letras en cada renglón en las últimas líneas ${ }^{11}$. 

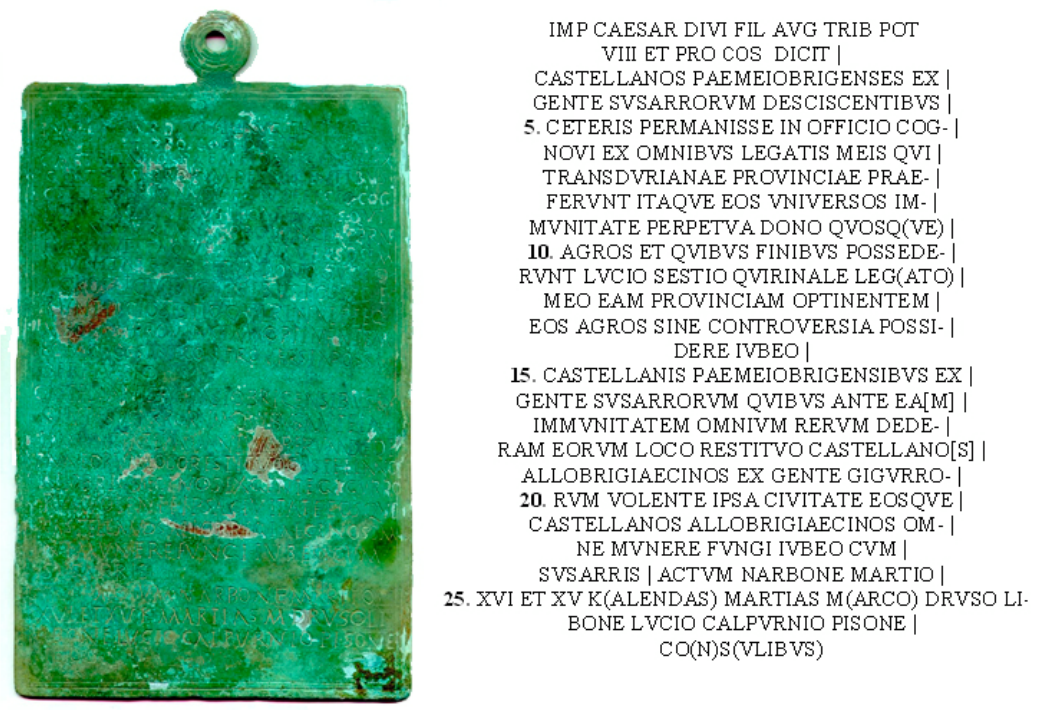

Fig. I. Bronce de Bembibre ${ }^{12}$

Las medidas del epígrafe son 24,4 x 15,3 x 0,2 cm., con unas letras de $0,65 \mathrm{~cm}$. de tipo capital con un ductus tendente a la uncialidad y un peso de 336 gr. En la actualidad se encuentra conservado en el Museo de León.

Se ha considerado que el epígrafe es una copia de un documento publicado en soporte más sencillo ${ }^{13}$, quizás una tabula cerata, que posteriormente sería realizado en bronce. Visualmente se puede observar la división del texto en cuatro partes, estando éstas compuestas por una introducción, un primer edicto, segundo edicto y la parte final, donde se indica el lugar de expedición y la fecha. En cuanto al contexto histórico, debemos ubicarlo en la reorganización del territorio noroeste peninsular por parte de Octavio Augusto, una vez finalizadas las guerras cántabras.

Pasando a un análisis interno del epígrafe, lo primero en considerar es el tipo de documento con el que nos encontramos, ya que los epígrafes romanos llevan en general una formula común en los documentos de una misma clase. En este caso se trata de un epígrafe con carácter jurídico-legal, ya que contiene edictos oficiales. El edicto presenta dos dataciones, una realizada en la introducción del texto que señala la octava potestad tribunicia de Augusto, lo cual es considerado una errata en opinión de diversos autores, que consideran que se trataría realmente de la novena ${ }^{14}$. Esta opinión se fundamenta en la comparación con la segunda datación realizada en la parte final del documento, que lo dataría en los días 14 y 15 de Febrero del año 15 
a.C.

El primer edicto hace mención a una provincia Transduriana, desconocida hasta el descubrimiento del documento, que debería englobar los territorios situados al Norte del Duero. La mención al legado L. Sextio Quirinal, ha hecho pensar que esta provincia pudo comenzar a existir entre el 22/19 a.C. y desapareció entre los años 15/13 a.C. a causa de la reorganización provincial llevada acabo por la administración romana en esta fecha ${ }^{15}$. Sin embargo, otras interpretaciones exponen que dicha provincia sería en realidad una administración territorial situada dentro de la provincia Tarraconense ${ }^{16}$.

En cuanto al motivo del primer edicto, hace referencia a la concesión de una inmunitas perpetua a la comunidad peregrina de los Paemeiobrigenses, como resultado de la fidelidad de esta comunidad hacia Roma, así como la corroboración de la extensión de sus tierras. El segundo edicto, señala que los Allobrigiaecini sustituyeran a los Paemeiobrigenses, cumpliendo las obligaciones que estos tenían anteriormente con Roma, y de las que habían sido liberados. De estos edictos podemos extraer diversos aspectos sobre el tipo de organización socio-política que poseía el área astur en castella, que se consideraba exclusiva del área de la Gallaecia, no existiendo contradicción entre la organización en gentes y castella, desde el momento que se testimonian unos castellani Paemeiobrigenses ex gente Susarrorum y otros castellani Aliobrigaecini ex gente Gigurrorum, todos ellos astures ${ }^{17}$.

Se observa también, gracias a este documento, el tipo de reorganización espacial que Augusto impondrá en esta zona, así como el trato dado a las comunidades indígenas, trazando unos límites territoriales, junto a los cuales fijará unas unidades fiscales para los territorios conquistados, y premiando y castigando a las distintas comunidades, según su trato hacia Roma.

\section{FUENTES NUMISMÁTICAS}

La numismática es particularmente útil en investigaciones de Historia Antigua, ya que se compone de restos arqueológicos en los que el hombre ha grabado sus ideas dominantes, revelando el carácter, las costumbres y las vicisitudes históricas que tales objetos nos dejaron, y sirviendo además como criterio de datación para la Arqueología. Dentro del amplio catálogo de acuñaciones con que se cuenta para este periodo, nos centraremos en las celtibéricas, particularmente en un semis (fig. II), en cuyo reverso aparece la imagen de un gallo, lo cual nos llama la atención, ya que dentro de los reversos de la amonedación celtibérica estamos acostumbrados a ver la clásica imagen del jinete a caballo con lanza. Vamos a realizar un pequeño análisis de dicha moneda, mediante el cual intentaremos repasar toda la información que estos restos materiales nos aportan, así como recalcar la complicada tarea 

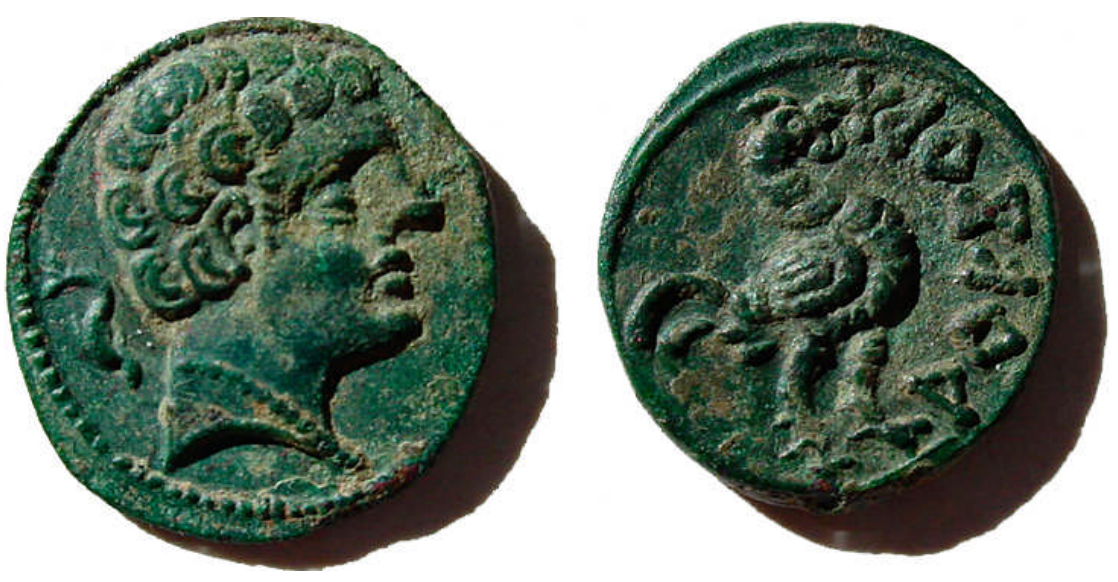

Fig. II. Semis de Arekoratas.

que supone su estudio e interpretación.

El semis, en cuestión, fue encontrado en los alrededores del pantano de la Yesa, desconociéndose el lugar exacto de su deposición, estando por ello descontextualizada arqueológicamente. El buen estado en que conserva sirve para deducir que debió estar poco tiempo en circulación. Posee un peso de 3,20 gr. y un módulo de 19 y 17,2 mm. En su anverso, podemos observar una cabeza imberbe con peinado de medios círculos concéntricos alineados en tres filas, con collar de línea seguida y orientada hacia la derecha, acompañada por un delfín. En el reverso aparece un gallo erguido de perfil hacia la derecha y una leyenda que se acomoda hacia la curvatura del cospel en la que está escrita en lengua celtíbera el nombre de la ceca, AREKORATAS.

En la antigüedad, cuando una población utilizaba una determinada imagen no lo hacía de forma aleatoria. Detrás de ese símbolo existía una serie de creencias tanto culturales como religiosas, que por algún motivo ligaban a esa imagen con el pueblo que la acuñaba, que se sentía representado con la misma. Para conocer el significado y motivo de la simbología entran en juego multitud de factores e influencias de tipo cultural, ritual, religioso, económico, social o histórico, responsables de la elección de una determinada imagen.

Comenzando con el anverso, se puede identificar la cabeza como la de Mercurio o Melkart (Hércules fenicio), que es la más acuñada en las monedas prerromanas y podría estar relacionada con aspectos religiosos e ideológicos de influencias de colonias hispano-cartaginesas ${ }^{18}$. El delfín por su parte, se relaciona con los viajes marítimos de Hércules, así como es también posible 
su vinculación a idea de la luz, relativo al culto de Apolo, con el que los griegos vinculan también a este animal marino ${ }^{19}$. Pero lo que particularmente llama la atención en esta moneda, como dijimos antes, es su reverso, donde se lee el nombre de la ceca: arekoratas, que posee un gran repertorio de monedas celtibéricas, para la cual no está fija su ubicación, siendo consecuencia del gran número de hallazgos situarla en los alrededores de la actual Ágreda (Soria). En tal repertorio se observa como está reservado para denarios y ases la clásica imagen del jinete a caballo con lanza en el reverso, mientras que para los semises se utiliza una imagen tan poco común como la del gallo, única además en las cecas celtibéricas. Pero, ¿por qué utilizaron esta imagen y qué representaba este motivo en concreto para la población de la ceca?

En distintas clasificaciones numismáticas de comienzos del siglo pasado, se señalaron ya las similitudes entre esta figura y las grabadas en divisores griegos atribuidas a sextantes de Emporiae y $\mathrm{Kese}^{20}$, con lo que podríamos considerar que esta imagen habría sido incorporada al numerario de la ceca como influencia de las poblaciones mediterráneas. Chaves y Marín, en está misma línea, consideraron que los tipos fueron copiados, pero que la elección de los mismos por parte de una comunidad tenía un significado especial, realizando para su copia una elección entre un gran numero de imágenes, desconociendo los motivos que hicieron decantarse por la del gallo' ${ }^{21}$. Otros autores como Domínguez Arranz lo relacionó con los ritos funerarios, al considerar al gallo, junto al caballo y el perro, como los animales que más aparecen en los enterramientos, y al suponer la sustitución que aquí hace el gallo respecto al animal equino ${ }^{22}$.

Por último, resaltar la importancia que posee la distribución espacial de los hallazgos de las monedas de la ceca, puesto que conocer las posibles relaciones de esta comunidad con otros territorios, quizás nos pueda también conceder nuevas interpretaciones en cuanto a su simbología.

\section{FUENTES ARQUEOLÓGICAS}

La Arqueología es una fuente de gran importancia para la investigación del periodo de romanización del interior peninsular. En esta etapa histórica se trabaja fundamentalmente con fuentes escritas procedentes del ámbito romano, poseyendo, del indígena, diversos epígrafes que nos aportan gran información, pero que no son muy numerosos, así como el nombre de las cecas escritas en la leyenda de las monedas indígenas. En cuanto a las fuentes literarias, no tienen un punto de vista imparcial de los hechos que cuentan, como hemos podido ver anteriormente. Se ocupan, en su mayoría, de hechos bélicos que atañen a Roma y de los cuales es difícil distinguir aspectos sociales, económicos o religiosos sobre los pueblos del interior. Los autores clásicos 
se olvidarán de la península una vez concluida la toma de Numancia. Tras la llegada de diez senadores romanos, para organizar los territorios, no volveremos a tener noticias sobre Hispania, excepto en puntuales episodios bélicos, hasta las guerras sertorianas y las guerras civiles entre Cesar y Pompeyo. Por lo que a la epigrafía romana se refiere, la escasa cantidad de documentos de época republicana, nos obliga en general a trabajar con documentos de la etapa imperial, para la que tenemos un gran repertorio, y que permite observar los cambios que se han sucedido como consecuencia del proceso romanizador, pero no de cómo se desarrollaron tales transformaciones. Nos encontramos, por tanto, ante un vacío documental, para casi más de sesenta años, que van desde la toma de la ciudad celtibérica a las guerras sertorianas, en los cuales se irán sucediendo multitud de cambios en la Meseta, resultado de la fusión de aspectos indígenas y romanos.

Una de las fuentes que permite despejar esta espesa niebla cronológica es la Arqueología. Las fuentes arqueológicas nos permiten vislumbrar a través de los restos materiales, aunque no sin dificultades, determinados cambios económicos, sociales, religiosos y políticos que tendrán lugar en este periodo. Un tipo de restos que proporciona gran información para conocer las transformaciones acaecidas en esta etapa son las necrópolis y, en particular, los ajuares funerarios.

Nos vamos a ubicar para este apartado, en el poblado vacceo-romano de Pintia, donde se encuentra uno de los espacios funerarios más importante, excavado a día de hoy en la Meseta Norte, constituido por la necrópolis vacceo-romana de las Ruedas. Dicho yacimiento posee una extensión de unas cuatro hectáreas, que responde a una ocupación radial, que fue usada como necrópolis, desde el siglo IV a.C. al final del I d.C. El ritual funerario que observamos en este cementerio, consistió en la combustión de los cuerpos de los fallecidos junto a su ajuar personal, para, posteriormente, ser depositados en una urna cineraria, y enterrados en hoyos junto a otras ofrendas, como alimentos y bebidas contenidos en diferentes recipientes. El estudio del cementerio ha permitido reconstruir aspectos de la posible reorganización sociopolítica del poblado, ya que los tipos de ajuares y ofrendas aparecidos en cada una de las tumbas, podrían representar el distinto rango por edad, sexo o condición, que jugó la persona en la comunidad. Aunque cabe destacar que, por muy reveladores que parezcan, dichos datos deben ser usados con cautela, ya que nos pueden llevar a diversos equívocos, como ha ocurrido en más de una ocasión, en otros yacimientos de tipo funerario ${ }^{23}$.

Basándonos en la información contrastada que aporta este yacimiento, podemos señalar que durante el proceso de romanización se producirá una adaptación y fusión de aspectos indígenas y romanos en los enterramientos. A los hallazgos de estelas con nombres latinizados ${ }^{24}$, se añade una miniaturización de los ajuares, así como la menor importancia que se dará a los restos 
óseos en el conjunto funerario ${ }^{25}$. Nos vamos a encontrar, para este periodo con restos arqueológicos que muestran la pervivencia de materiales y ritos indígenas, tales como cerámicas pintadas torneadas y sacrificios de animales domésticos, que se fusionarán con una adopción progresiva de gustos romanos por parte de la élite vaccea. Lo pone de manifiesto, hallazgos tales, como la presencia de cuencos de terra sigillatta y lampadarios, o la detección, dentro del cuenco de vidrio, de un vino al gusto romano, amielado y aromatizado con rosáceas ${ }^{26}$.

\section{ASPECTOS BIBLIOGRÁFICOS}

Por último, queremos destacar algunos recursos que pueden resultar muy beneficiosos a la hora de buscar información sobre este periodo, con el objetivo de facilitar el trabajo a cualquiera que le interese el tema.

$\mathrm{El}$ primer contacto que tiene un tesinando con su tema de su investigación esta marcado por la enorme recopilación bibliográfica que deberá realizar durante los primeros meses de su trabajo, seleccionando y desechando gran cantidad de artículos, libros y demás recursos. El recurso fundamental que va a poseer el futuro investigador en esta primera etapa será su propio director de tesina, que le irá orientando sobre los diferentes materiales que le permitirán comenzar su investigación, así como los lugares donde poder encontrarlos. Pero, a medida que nos introduzcamos en el tema, iremos abriendo también propios campos de búsqueda. Para ello puede resultar útil consultar diferentes manuales y revistas científicas que estén relacionadas con el periodo a tratar, teniendo a nuestra disposición gran cantidad de artículos referidos a Arqueología e Historia Antigua ${ }^{27}$, así como un gran número de publicaciones sobre aspectos relacionados con el tema, que se pueden encontrar gracias a la multitud de recursos electrónicos y bases de datos bibliográficos con las que contamos en la actualidad ${ }^{28}$.

\section{Notas}

${ }^{1}$ Nepote: Hamilcar 4, 2; De viris illustribus 52.

${ }^{2}$ Livio. 35, 7, 8; Liv. 35, 22, 8.

${ }^{3}$ Caes. b. e. I, 38, $1-4$.

${ }^{4}$ Estrabón. III, 3, 5.

${ }^{5}$ Estrabón. III, 4, 16: “Se cuenta que los vettones la primera vez que llegaron a un campamento romano, viendo a algunos centuriones ir y venir paseando por entretenimiento, lo tuvieron por cosa de locos y les enseñaron el camino hacia sus tiendas considerando que bay que dedicarse a reposar, echados tranquilamente, o 
combatir".

${ }^{6}$ Lucano. IV. 4-1: "Con éstos estaban aqui, además de las armas latinas, el activo astur, los ligeros vettones, los celtas exiliados del antiguo pueblo galo y mezclando su nombre al de los iberos".

${ }^{7}$ Plinio, Nat. Hist. III, 19: "yendo hacia el interior (...) los Mensetanos, los Oretanos y, junto al Tajo, los Carpetanos; junto a ellos los Vacceos, Vettones y Celtíberos Arévacos."

Plinio, Nat. Hist. IV, 116: "Sus gentes son los célticos, los túrdulos, junto al Tajo los Vettones y desde el Ana hasta el Sacrum los Lusitanos"

${ }^{8}$ Plinio, Nat. Hist. IV, 112: "el Duero, río de los más grandes de Hispania, nacido en la región de los pelendones, corre junto a Numancia, luego a través de los arévacos y vacceos y separa a los vettones de Asturia, de Lusitania a los galaicos, y marca alli todavía los límites entre los túrdulos y los brácaros".

9 O. López Jiménez, 'Las fuentes antiguas y la creación literaria de la Vetonia”, Gerión, 22, 2004, p. 206.

${ }^{10}$ J. M. Roldán Hervás, "Fuentes Antiguas para el estudio de los Vettones”, Zephyrus, 19, 1968, p. 2.

${ }^{11}$ G. Alföldy, "El nuevo edicto de El Bierzo en Hispania”, en L. Grau Lobo y J. L. Hoyas (eds.), El bronce de Bembibre, un edicto del Emperador Augusto del año 15 a.C., Museo de León, Junta de Castilla y León, Valladolid, 2001, p. 21.

${ }^{12}$ (fig.1) Texto traducido: "El emperador César Augusto, bijo del divino, en su octavo poder tribunicio y procónsul, dice: Conocí por todos mis legados que presidieron la provincia Transduriana, que los del castro de Paemeiobrigense, de la gens de los Susarros, se habian mantenido fieles, mientras que los demás eran disidentes. Por ello, a todos ellos les hago donación de inmunidad perpetua y ordeno que posean sin discusión las tierras, y en los mismos límites que poseyeron cuando mi legado Lucio Sestio Quirinale gobernó esta provincia. A los del castro Paemeiobrigense, de las gens de los Susarros, a los que les condedí inmunidad total, reintegro a su asentamiento a los del castro Allobrigiaecino, de la gens de los Gigurros, con asentamiento en la misma ciudad, y ordeno que estos del castro Allobrigiaecino cumplan todas sus obligaciones junto con los Susarros.Dado en Narbona Martio en los días 16 y 15 antes de kalendas de marzo, siendo cónsules $M$. Druso Libón y Lucio Calpurnio Pisón”.

${ }^{13}$ G. Alföldy, op. cit., p. 19.

${ }^{14}$ J. Gómez Pantoja y F. Martín González, "Notas sobre el edicto del Bierzo a la luz de otras constituciones de Augusto", en F..J Sánchez-Palencia y J. Mangas (eds.), El Edicto del Bierzo y la primera organización del Noroeste por Augusto, Fundación Las Médulas, León, 2001, pp.123135.

${ }^{15}$ M. Cavada Nieto y M. Villanueva Acuña, "El edicto de Bembibre y las reformas administrativas de Augusto en el noroeste”, en L. Grau Lobo y J. L. Hoyas (eds.), El bronce de Bembibre, un edicto del Emperador Augusto, Museo de León, Junta de Castilla y León, Valladolid, 2001, p.130.

${ }^{16} \mathrm{G}$. Alföldy, op. cit., pp.22-23.

${ }^{17}$ J. Mangas, "Castellum, gens, y ciuitas en el Edicto de Augusto (15 a. C.)”, en F. J. Sán- 
chez-Palencia y J. Mangas (eds.), El Edicto del Bierzo y la primera organización del Noroeste por Augusto, Fundación Las Médulas, León, 2001, pp. 47-60.

${ }^{18}$ M. A. Domínguez Arranz, "La moneda celtibérica”, Celtas y Vettones, Torreón de los Guzmanes, Iglesia de Santo Tomé el Viejo, Ávila, Septiembre-Diciembre 2001, pp. 218-227.

${ }^{19}$ R.Guénon, Simbolos fundamentales de la ciencia sagrada, Paidós Ibérica, Barcelona, 1995, p. 134.

${ }^{20}$ A. Delgado, Nuevo método de clasificación de las medallas autónomas de España, vol. III, Sevilla, 1876 , p. 23.

${ }^{21}$ F. Chaves, y M. C. Marín, "Numismática religiosa y religión romana en Hispania”, en La Religión Romana en Hispania (Simposio organizado por el Instituto de Arqueología Rodrigo Caro del C.S.I.C del 17 al 19 de diciembre de 1979.), Ministerio de Cultura, Madrid, 1981, pp. 27 y ss.

${ }^{22}$ M. A. Domínguez Arranz, "Nuevos hallazgos de bronces con leyenda celtibérica Arekorata", Bolskan: Revista de arqueología del Instituto de Estudios Altoaragoneses, 5, 1988, p. 258.

${ }^{23} \mathrm{H}$. Härke, "Warriorg Raves? The Background of the Anglo-Saxon Weapon Burial Rite", Past and Present 126, 1990, pp. 22-43.

${ }^{24}$ C. Sanz Mínguez, "Las Ruedas de Pintia: Nuevos datos para la contextualización de las estelas funerarias discordes”, en C. Sanz Míngues y J. Velasco Vázquez, (eds.), Pintia, un "oppidum" en los confines orientales de la región vaccea: investigaciones arqueológicas vacceas, romanas y visigodas (1999-2003), Universidad de Valladolid, Valladolid, p. 203.

${ }^{25}$ C. Sanz Mínguez, op. cit., p. 210.

${ }^{26}$ Tumbas 67 y 68 de la Necrópolis de las Ruedas (Pintia),, en C. Sanz Mínguez, op. cit., pp. 212-220.

${ }^{27}$ Listado de diversas revistas científicas que nos aportan gran información sobre los temas tratados en este trabajo: Studia Histórica; Gerión; Zephyrus; Paleohispánica; Historia Antiqua; GaIlaecia; RevArq; Conimbriga ; Veleia; Past and Present; Florilegium; Lancia; Numisma.

${ }^{28}$ Bases de datos donde buscar referencias bibliográficas sobre los temas tratados: Isoc; Historical abstract; ICYT; Dialnet; Sabus-Usal; Institute of Historical Research; TOCS -IN; Compludoc; Classical Resources , Diccionario topográfico de la Roma antigua; Epigraphische Datenbank. Heidelberg; Archaeology and Architecture; Archivo Epigráfico de Hispania; CEIPAC. 